\title{
WAPA: A wearable framework for aerobatic pilot aid
}

\author{
Xavier Righetti ,Sylvain Cardin, Daniel Thalmann, \\ VRLAB, EPFL, Lausanne, Switzerland \\ \{Xavier.Righetti, Sylvain.Cardin, Daniel.Thalmann\}@epfl.ch
}

\begin{abstract}
Disorientation induced by G-forces during aerobatic flight generates difficulties for the pilots to perfectly align their aerobatic maneuver. This paper presents a modular wearable system for enhancing training of aerobatic pilots. A combination of accelerometers and a gyroscope is used to detect possible deviations compared to the optimum trajectory. The wearable system informs the user in real time about the corrections to apply via vibrotactile actuators and speech synthesis. This publication presents a work in progress in order to validate the system in simulation.
\end{abstract}

Keywords: wearables, user interaction, task guidance, vibrotactile, accelerometer

\section{Introduction}

Aerobatic flying pushes the pilot biological conditions to its limit. The main exercise in aerobatic flight is to align perfectly the airplane during the various imposed maneuvers. G-forces induced by the movement of the airplane create vestibular dysfunction [1]. The vestibular apparatus is responsible with proprioception for self representation for our body orientation in space. Deficiency of the sensory system under such circumstances leads to errors in trajectories and in severe cases to accidents.

In demanding situations such as piloting a plane, the user should focus at $100 \%$ on his task and thus cannot be distracted by displays and other information outputs. The use of wearable devices is especially tailored for this kind of applications where the user's hands, eyes and attention are actively engaged with the physical environment.

In this paper, we will present an approach in which the user will select the devices that he wants to wear depending on the functionalities he wants to use. We focus on the present publication in our work in progress using wearable intelligent clothing to provide extra sensory information for aerobatic pilots.

Next section will describe the current state of the art concerning the use of wearables in task intensive situations, while section three will introduce our proposition. Section 4 will describe the setup of our experiments and finally, we will present our conclusions and remarks about future work in the last section. 


\section{State of the Art}

Different research has been performed using vibrotactile stimulation to improve self orientation in different environment. Some research focuses in altered gravity environment like heavy and 0 G-force for pilot and astronaut [2] [3]. Those researches show improvement of the mental representation of the body orientation in space. The use of Pneumatic and electromagnetic actuators located in a vest to give directional information about the position of different targets has been tested for correcting drift during aircraft piloting [4]. The conclusion of this research was very positive. Feedback from the test pilots shows that this form of tactile feedback reduces drastically the attention need for presented parameters; also it is intuitive and accurate. Measurement of trajectory error and response time between the use of the TSAS and an additional visual indicator shows clear improvement using tactile feedback over visual display.

Joined research, done at the department of experimental psychology at the University of Oxford and the Transport Research Laboratory of Wokingham, studies the influence of vibrotactile collision warning signals in driving simulation [5]. Their setup was using two vibration actuators fixed on the user belt, one in front and one in the back. The driver also has a three-state visual indicator to show if he is too close, too far or just at a good distance from the car ahead. The trial is to follow a car which will accelerate and brake at random time with disabled brake lights. This experiment shows a great improvement in response time while using the vibrotactile.

Many researches have shown the potential of attaching accelerometers for sensing body motion. Typical applications include activity recognition [6] [7], gesture recognition [8], medical rehabilitation [9] and ambulatory monitoring [10]. Alternately, MIThril, a complete wearable platform [11], has been developed to facilitate the development of distributed real-time multimodal and context-aware applications. Used in conjunction with the WearARM [12], a wearable computing core, researchers are trying to build a high-performance wearable system that is truly wearable and unobtrusive. For every wearable implementation, one must always choose a tradeoff between bulkiness and performance. In our approach, we favored the "wearability" of the system over the processing power, ruling out specific applications such as mixed reality guidance where the use of computer vision requires intensive calculations. This kind of application can be seen in [13], where the authors

have investigated the use of wearable computers to provide task guidance in aircraft inspection. In the preliminary results, they noticed that the user tends to over-rely on the computer and that the user's performance is directly related to the design quality of the user interface.

\section{Proposition}

As we have seen in the previous section, various garments with embedded computing devices input and output systems have been developed but they are usually made for one purpose. In our case, we have envisioned to build a versatile jacket with an embedded I2C bus (cf. Figure 1) and magnetic connectors in order to let the user 
keep the same garment and choose the desired functionalities according to the modules that will be attached to it.

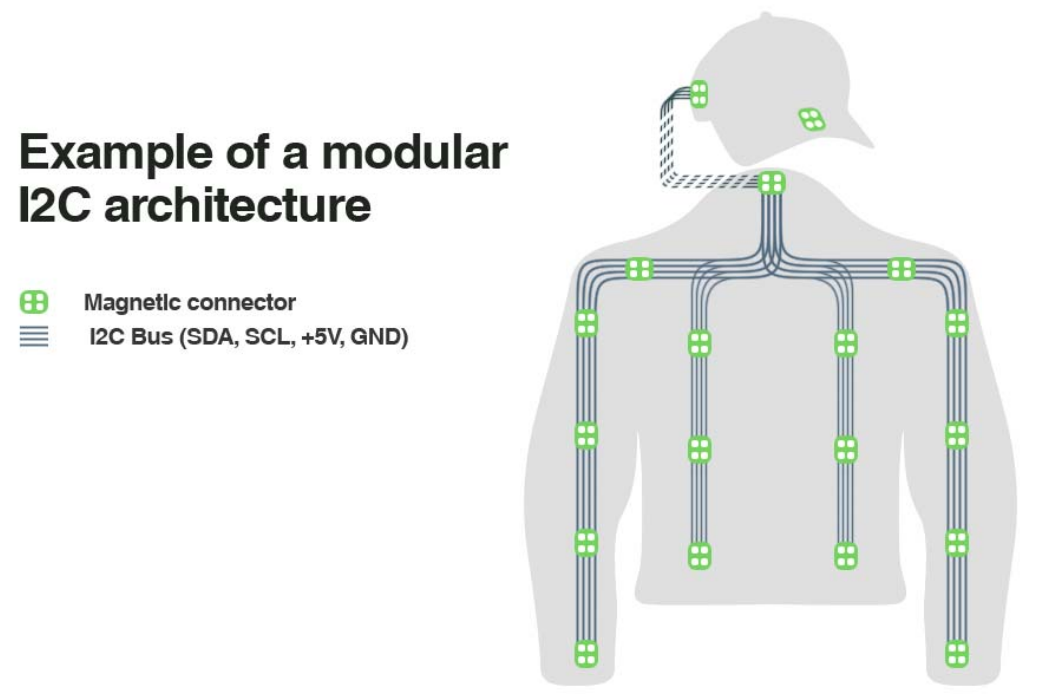

Figure 1: Schematic view of the I2C bus embedded into a jacket

In this case, the jacket will be worn by an aerobatic pilot during the flight in order to help him improve the perfect achievement of aerial maneuvers. Therefore, sensors like gyroscopes, compasses and accelerometers will be used to detect the plane's attitude while actuators like vibrotactile devices and speakers will inform the user about eventual corrections to take into account. The communication between the pilot and his/her wearable system is achieved in such a way that neither hands nor the eyes are distracted from the piloting task.

\subsection{Human-machine interface scenario}

The user is in control of the aircraft at a sufficient altitude and the area is clear of other aircraft or obstacles. The pilot wears our system embedded into his jacket and follows a straight path with no significant wind.

The pilot selects the aerobatic maneuver to perform by selecting onto the touch screen the appropriate icon located on his left forearm. The icon represents a standard perfect maneuver, roll or loop, computed accordingly with the current aircraft.

The text to speech module confirms orally which maneuver has been chosen and start a count down from ten seconds. At the end of the countdown the pilot should start while the text to speech module will indicates the angle at which the aircraft should be all along the realization of the maneuver.

The vibrotactile system will indicates the error between its current orientation and the predicted orientation using the prerecorded standard maneuvers as comparison. 
The tactile system will stimulate the user upper body skin by localized vibrations. A vibration on the left will indicated that the user should roll more on its left, one on is back indicates that he should pitch more up. The intensity of vibration will match the size of the deviation error.

\section{Setup}

We have designed our system in such a way that all wearable modules are connected together using the two wires I2C protocol. $\mathrm{I}^{2} \mathrm{C}$ (Inter-Integrated Circuit) is a simple half-duplex1 serial 2-wire bus developed by Philips in the early 1980's for efficient communication between different ICs (Integrated Circuits). It has been designed originally to solve many interfacing problems such as synchronization issues when designing digital control circuits and has now become a world standard. In our implementation, the I2C bus is composed of four wires: two for transmitting data (clock signal and data signal) and two for powering the modules with a $5 \mathrm{~V}$ voltage. The connectors for attaching the modules are made of a simple flexible PCB, as seen on Figure 2. On top of it, four magnets are glued with a silver epoxy conductive glue. The magnets are arranged in a way that they will repel the module if the user tries to attach it the wrong way.
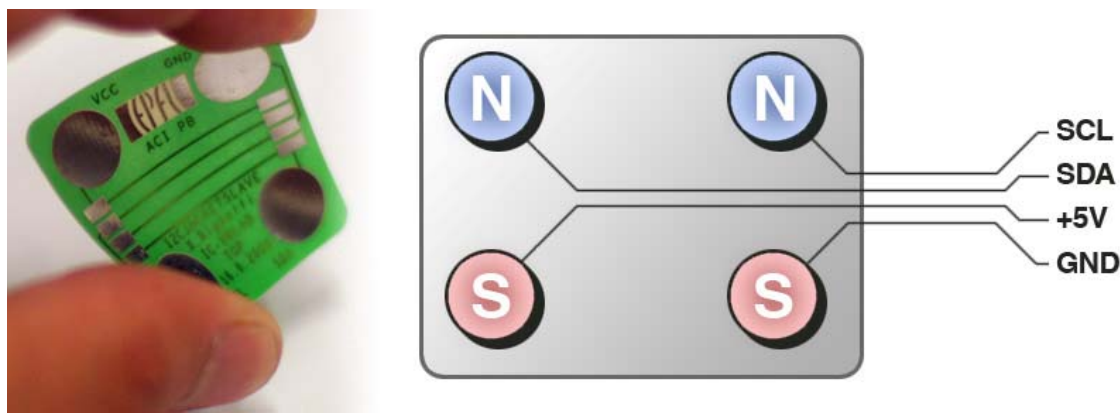

Figure 2: Flexible connector and magnets arrangement

\subsection{Master Module}

The master module will be responsible for managing the communication for all slave modules, centralizing the data coming from various sensors, processing them and controlling the embedded actuators such as the vibrotactile modules and the textto-speech module. It is composed of an 8bit microcontroller (PIC18F8722), a Bluetooth adapter (Bluegiga WT11) and an I2C driver. Additionally, an attached oled

\footnotetext{
${ }^{1}$ transmission of information in opposite directions but not simultaneously
} 
tactile display will help the pilot during the pre-flight situation to calibrate the modules and to select the maneuver he/she wants to train.

\subsection{Slave orientation tracker module}

We plan to use the MTx sensor from Xsens, which is a small and accurate 3DOF Orientation Tracker. It provides drift-free 3D orientation as well as kinematic data: 3D acceleration, 3D rate of turn and 3D earth-magnetic field. This sensor will help us determine the deviation error according acceleration values calculated for the optimal trajectory in simulation.

\subsection{Slave text-to-speech module}

The text-to-speech module will announce the name of the maneuver to practice and when to start it. Moreover, it will inform the user about his error rate at the end of the maneuver. The module will be connected with the onboard headphone system and thus will improve the interaction with the user by giving auditory feedbacks.

\subsection{Slave vibrotactile modules}

A combination of 32 vibrotactile modules will help the pilot correcting his trajectory in real time by applying vibrations on the body. The position and the strength of the vibration will inform the user about the eventual deviation compared to the optimal trajectory and thus about the kind of correction that has to be applied.

\section{Experiment and validation}

In order to validate our experiment we used a simulation setup around the flight simulator X-plane ${ }^{2}$, as seen on Figure 3. The simulator offers realistic airplane behavior and coherent piloting condition using a throttle box and a joystick. Although this setup will not be sufficient to assess the full efficiency of the current system due to the lack of G-forces and stress pressure induced in the real situation. This system has been considered sufficient to provide a validation platform for test and calibration of the final system.

We are using our embedded system connected to the flight simulator. The main difference is that the value from the Xsens input sensor is recovered from the simulator instead of the real sensor.

\footnotetext{
${ }^{2}$ www.x-plane.com
} 

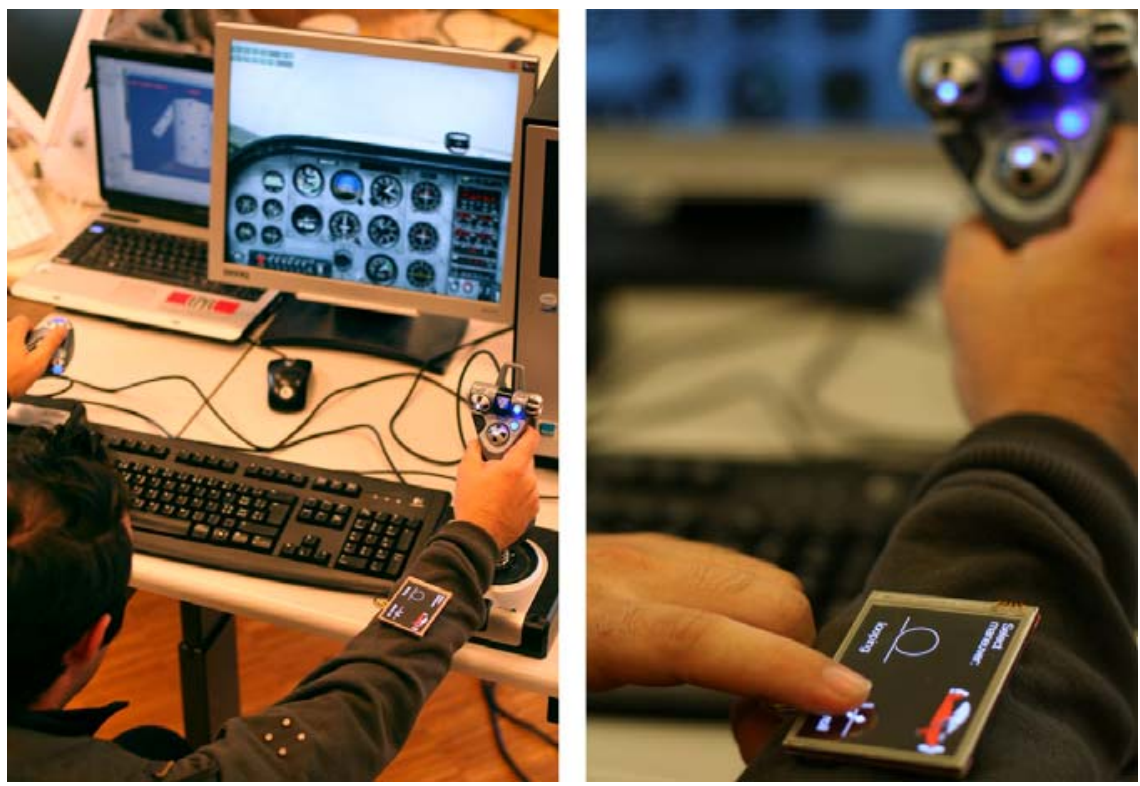

Figure 3: Simulation tests

Xplane allows development of custom plug-in. We implemented a UDP server module to recover the different orientation description. We mainly used the euler angles values and their derivative. We compare them between a "perfect maneuver" and the current airplane behavior.

In order to find the best place to put the vibration actuator module we used a vibrotactile jacket developed during previous researched [ref]. This jacket includes 32 vibration motors from mobile phone technology (cf. Figure 4). The jacket will be connected as slave module inside the wearable platform in the same manner that the single vibration actuator module.

The system will also be connected to a computer which will control the simulation, recover the orientation and trajectory of the aircraft, and generate accordingly the vibration feedback. It also includes a calibration interface for the vibrotactile actuator. This external device is communicated wireless using Bluetooth module with the master module of the wearable system. This system is there only to tune up the vibration feedback in simulation. For the final setup, the whole feedback generation algorithm will be embedded inside the slave vibration modules. 


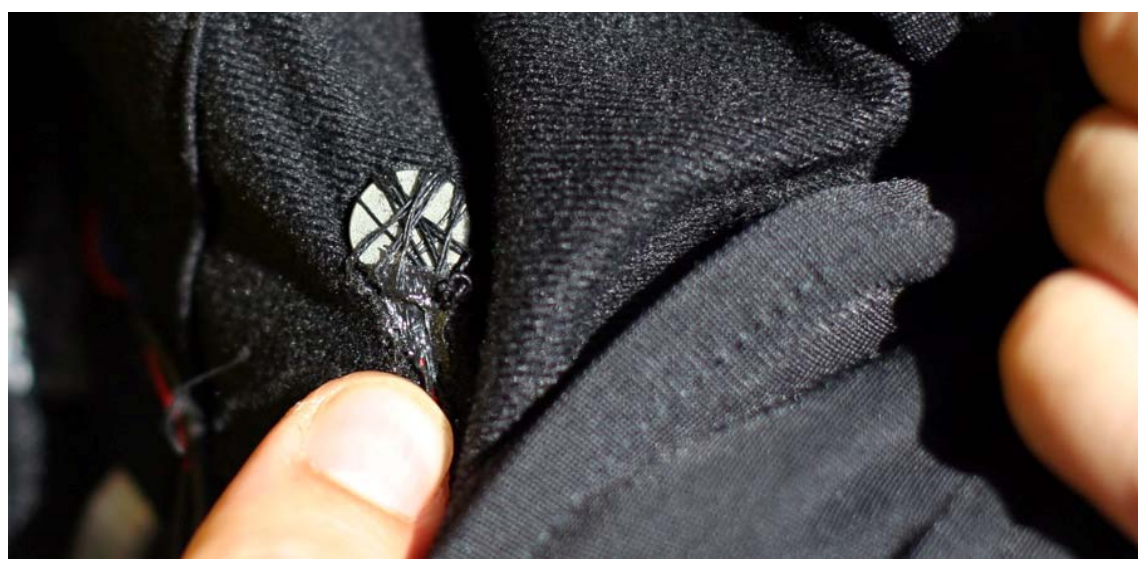

Figure 4: One of the 32 vibration motor embedded in the jacket

\section{Test procedure:}

In order to validate our system we will use a sample population of persons who will fly an aerobatic aircrafts inside the simulator. We will assume that every test subject will be able to perform the two recommended basic maneuver which will consist in a roll and a loop.

That assumption is really delicate since the performance is linked to previous piloting experience. We will let user getting familiarized with the simulator for sufficient time for them to be able to keep a direction and perform the required basic moves. It could require from a few minutes to several hours depending of the subject.

To allow comparison of the influence of the wearable system from one user to another, only the difference between trajectories using or not using the tactile feedback is being used to determine its efficiency. The error difference to an established standard and perfect maneuver reflects the ability of the subject to perform the task. If we demonstrate that the performance is improved by the use of the wearable device on a sufficiently large population we can distinguish the impact of such technologies in extreme condition.

User acceptance is also a crucial question when you want to access the impact of Human machine interface technology. In order to recover user feedback we have developed a small questionnaire concerning their background concerning piloting task, their confidence and feeling about the wearable system. We focus questions on self representation of the aircraft behavior, vibrotactile feedback coherence, overall system comfort and user interest in the system.

\section{Early results and future work}

We have performed the experience with a few intern subjects in order to tune up the different parameters of the simulation. The first results are encouraging. We detect slightly better performances while using vibrotactile feedback. The main issue we 
encountered is the difficulty to perform loops and rolls correctly without much training on the simulator. The system is efficient for small trajectory correction but if the plane is getting really off axis it becomes difficult to get coherent feedback.

We obtain a fully functional simulation platform and processed to overall calibration of the system.

The next step is to contact and get feedback from real aerobatic pilots, fix the aircraft model and refined vibrotactile pattern in order to embed complete vibration generation. Perform large study in simulation to validate properly our proposition. The final goal will be to test this system in real aerobatic situation and perform live experimentation.

\section{Acknowledgments.}

The work presented was supported by the EU project INTERMEDIA (38419), in the frame of the EU IST FP6 Programme.

\section{References}

1. Muller TU: G-induced vestibular dysfunction (“the wobblies”) among aerobatic pilots: a case report and review, in: Ear Nose Throat,pp:269-272 (2002)

2. Traylor, R. and Tan, H. Z. 2002 : Development of a Wearable Haptic Display for Situation Awareness in Altered-gravity Environment: Some Initial Findings. In: Proceedings of the 10th Symposium on Haptic interfaces For Virtual Environment and Teleoperator Systems HAPTICS. IEEE Computer Society, Washington, DC, pp: 159 (2002)

3. van Erp, J.B.F. and Van Veen, H.A.H.C. A multipurpose tactile vest for astronauts in the international space station., In :Proc. Eurohaptics 2003, pp: 405-408 (2003)

4. Raj, A. K., Suri, N., Braithwaite, M. G., and Rupert, A. H. :. The tactile situation awareness system in rotary wing aircraft: Flight test results. In : Proceedings of the RTA/HFM Symposium on Current Aeromedical Issues in Rotary Wing Operations. RTO NATO, Neuilly-sur-Seine, France, pp: 16.1-16.7 (1998)

5. Ho, C., Reed, N. J., \& Spence, C. ::Assessing the effectiveness of "intuitive” vibrotactile warning signals in preventing front-to-rear-end collisions in a driving simulator. In :Accidents Analyses and Prevention, 38, pp : 988-996 (2006).

6. Ermes, M., Parkka, J., Mantyjarvi, J., Korhonen, I: Detection of daily activities and sports with wearable sensors in controlled and uncontrolled conditions. In: IEEE Transactions on Information Technology in Biomedicine, vol. 12, pp. 20-26 (2008)

7. Van Laerhoven, K., Gellersen, H.W: Spine versus porcupine: A study in distributed wearable activity recognition. In: Proc. of the Eighth IEEE Intl. Symposium on Wearable Computers, vol. 1, pp. 142-149 (2004)

8. Cho, I., Sunwoo, J., Son, Y., Oh, M., Lee, C.: Development of a Single 3-axis Accelerometer Sensor Based Wearable Gesture Recognition Band. In: Lecture notes in Computer Science, Springer, vol. 4611, pp. 43--52 (2007)

9. Bonato, P.: Advances in wearable technology and applications in physical medicine and rehabilitation. In: Journal of NeuroEngineering and Rehabilitation, vol. 2, BioMed Central Ltd (2005)

10. Karantonis, D.M., Narayanan, M.R., Mathie, M., Lovell, N.H., Celler, B.G.: Implementation of a real-time human movement classifier using a triaxial accelerometer for 
ambulatory monitoring. In: IEEE transactions on information technology in biomedicine, vol. 10, pp. 156-167 (2006)

11. DeVaul, R., Sung, M., Gips, J., Pentland, A.: Mithril 2003: applications and architecture. In: Proceedings of the Seventh IEEE International Symposium on Wearable Computers, pp. 4--11 (2005)

13. Lukowicz, P., Anliker, U., Tröster, G., Schwartz, S.J., DeVaul, R.W.: The weararm modular, low-power computing core. In: IEEE micro, pp. 16--20 (2001)

12. Ockerman, J.J, Pritchett, A.R.: Preliminary Investigation of Wearable Computers for Task Guidance in Aircraft Inspection, In: Second International Symposium on Wearable Computers, pp. 33--40 (1998) 